\title{
Tratamento artroscópico da instabilidade anterior traumática do ombro: resultados a longo prazo e fatores de risco*
}

\author{
Arthroscopic treatment of traumatic anterior instability \\ of the shoulder: long term results and risk factors
}

\begin{abstract}
Glaydson Gomes Godinho', Flávio de Oliveira França², José Márcio Alves Freitas³, Cristiano Magalhães Menezes ${ }^{4}$, Saulo Garzedim Freire ${ }^{5}$, Andrey Leal Wanderley ${ }^{6}$, Luís Guilherme de Castro Santos ${ }^{7}$
\end{abstract}

\section{RESUMO}

Objetivo: Avaliar os resultados de longo prazo e fatores de risco no tratamento cirúrgico por via artroscópica da instabilidade anterior traumática do ombro. Métodos: Estudo retrospectivo de 302 pacientes (314 ombros). Seguimento de 24 a 140 meses, média de 76,5 meses. Avaliação através de exame físico e radiográfico. Classificação de resultados segundo os critérios da UCLA e Carter-Rowe. Aná-

Trabalho realizado no Hospital Ortopédico e Hospital Belo Horizonte, Belo Horizonte (MG), Brasil.

1. Doutor, Chefe do Grupo de Ombro do Hospital Ortopédico e Hospital Belo Horizonte, Belo Horizonte (MG), Brasil.

2. Cirurgião do Grupo de Ombro do Hospital Ortopédico, Belo Horizonte (MG), Brasil.

3. Cirurgião do Grupo de Ombro do Hospital Ortopédico e Hospital Belo Horizonte- Belo Horizonte (MG), Brasil.

4. Ortopedista do Corpo Clínico do Hospital Ortopédico, Belo Horizonte (MG), Brasil.

5. Residente do 4o ano, Hospital Ortopédico, Belo Horizonte (MG), Brasil.

6. Residente do 4o ano do Grupo de Ombro do Hospital Ortopédico, Belo Horizonte (MG), Brasil.

7. Residente do Grupo de Ombro do Hospital Ortopédico, Belo Horizonte (MG), Brasil.

Endereço para correspondência: Dr. Glaydson Gomes Godinho, Rua Professor Otávio Coelho Guimarães, 111, Mangabeiras - 30210-

300 - Belo Horizonte, MG. Fax: (31) 3227-0666.

E-mail: ggodinho@terra.com.br

Recebido em 18/3/08. Aprovado para publicação em 2/5/08.

Copyright RBO2008 lise estatística através de programa Epi Info e testes $t$ de Student e exato de Fisher. Resultados: Observado índice de recidivas em $8,9 \%$ dos ombros. Segundo a UCLA, excelentes/bons resultados observados em $97,6 \%$ e, regulares/ruins em $2,4 \%$ dos ombros. Segundo Rowe, excelentes/bons resultados em 89,2\% e regulares/ruins em $\mathbf{1 0 , 8 \%}$ dos ombros. Observada significância $(p=0,013)$ entre uso de materiais adequados, curva de aprendizado e índice de recidivas. História de convulsões $(p=0,0039)$ e prática de esportes de contato $(p=0,004)$ tiveram forte correlação com recidivas. Não se evidenciou correlação entre lesão de Bankart ( $p=0,546$ ), lesão de HillSachs $(p=0,62)$ e recidivas, considerando-se lesões ósseas menores que $25 \%$ da glenóide e cabeça umeral, respectivamente. Conclusões: 1) Há correlação estatisticamente identificada entre o índice de recidiva da instabilidade anterior traumática do ombro e: a) convulsões no pós-operatório; b) prática de esportes de contato; c) presença das lesões de Bankart com Hill-Sachs caracterizadas por $25 \%$ ou mais de lesão óssea da glenóide ou da cabeça umeral, respectivamente. 2) Parece haver correlação entre o não reparo da lesão $S L A P$ e o índice de recidiva da instabilidade anterior traumática do ombro.

Descritores - Artroscopia; Instabilidade articular/cirurgia; Articulação do ombro/cirurgia; Recidiva; Técnicas de sutura; Parafusos ósseos; Estudos retrospectivos 


\section{ABSTRACT}

Objective: To evaluate the long term results and risk factors in the surgical arthroscopic treatment of the traumatic anterior instability of the shoulder. Methods: Retrospective study of 302 patients (314 shoulders) 24 to 140 month follow-up, with a mean of 76.5 months. Evaluation performed in clinical and radiological exams. Results classified according to the UCLA and Carter-Rowe. Statistical analysis with the Epi Info program, T-student calculations, and Fisher Exact Test. Results: A rate of $8.9 \%$ of recurrence was seen in the shoulders. According to UCLA classification, excellent/good results were seen in $97.6 \%$, and regular/poor results, in $2.4 \%$ of the shoulders. According to Rowe classification, excellent/good results were seen in $89.2 \%$, and regular/poor results, in $10.8 \%$ of the shoulders. There was significance $(p=0.013)$ among the use of adequate materials, learning curve, and recurrence rate. A history of seizures $(p=0.0039)$ and the practice of contact sports $(p=0.004)$ had a marked correlation with recurrence. No correlation was seen between the Bankart lesion ( $p=0.546)$, the HillSachs lesion $(p=0,62)$ and recurrences, considering bone lesions smaller than $25 \%$ of the glenoid and humeral head, respectively. Conclusions: 1. There is a statistically identified correlation between the rate of recurrence of traumatic anterior stability of the shoulder and: a) seizures in the post-operative period; b) practice of contact sports; c) presence of Bankart lesions with Hill-Sachs characterized by $25 \%$ or more of bone lesion of the glenoid or of the humeral head, respectively. 2. There seems to be a correlation between the non-repair of the SLAP lesion and the recurrence rate of traumatic anterior stability of the shoulder.

\footnotetext{
Keywords - Artroscopy; Joint instability/surgery; Shoulder joint/surgery; Recurrence; Suture techniques; Bone screws; Retrospective studies
}

\section{INTRODUÇÃO}

A luxação primária anterior de ombro ocorre habitualmente em jovens durante esportes de contato ou por queda com trauma de baixa energia em idosos. A luxação anterior em idosos tem complicações peculiares, enquanto a instabilidade recidivante é um problema particular nos jovens.

Nas últimas três décadas houve grande avanço no desenvolvimento técnico da cirurgia artroscópica para tratamento das instabilidades do ombro. Contribuiu para o nível progressivo de excelência dos resultados a melhor seleção de pacientes, o aprimoramento das técnicas, a melhora na qualidade do instrumental cirúrgico e de implantes, além da experiência crescente dos cirurgiões.

Johnson propôs o reparo com grampos da lesão de Bankart, por via artroscópica, obtendo alto índice de recidivas e complicações como lesões osteocondrais em seus resultados ${ }^{(1)}$. Seguindo o mesmo princípio e com o objetivo de diminuir as recidivas, outros autores desenvolveram novas técnicas artroscópicas ${ }^{(2-4)}$. Morgan et al usaram fio de sutura absorvível transglenóideo para o reparo da lesão de Bankart ${ }^{(3)}$. Caspari et al também usaram técnicas de reparo transglenóideo por via artroscópica ${ }^{(5)}$. Carrera et al utilizaram a técnica transglenoidal com fio inabsorvível e ponto de sutura em "U", obtendo $20 \%$ de recidivas ${ }^{(6)}$. Godinho et al relataram o uso da técnica de Morgan, obtendo $13,9 \%$ de recidivas em 79 pacientes $^{(7)}$.

$\mathrm{O}$ surgimento da técnica de sutura com âncoras representou importante avanço no reparo das lesões labiais, na medida em que possibilitou a realização de toda a fixação da lesão de Bankart com pontos intra$\operatorname{articulares}^{(8-9)}$.

Burkhart et al relataram alto índice de recidivas póscirúrgicas em artroscopia para estabilização do ombro, quando presente significativa lesão óssea glenoidal $^{(10)}$.

O objetivo deste estudo é a avaliação retrospectiva e a análise dos fatores que influenciaram no resultado cirúrgico de pacientes submetidos ao tratamento artroscópico da instabilidade anterior glenoumeral, por meio do uso de miniâncoras metálicas e sutura. 


\section{MÉTODOS}

No período compreendido entre 25 de abril de 1996 e 24 de novembro de 2005, 344 pacientes foram submetidos ao tratamento cirúrgico por via artroscópica, para correção da instabilidade ântero-inferior traumática de ombro com seguimento mínimo de 24 meses, máximo de 140 e média de 76,5 meses. As cirurgias foram realizadas no Hospital Ortopédico e Hospital Belo Horizonte, em Belo Horizonte/MG. Todos os pacientes foram operados por cirurgiões do grupo de ombro dos referidos hospitais.

Foram excluídos 42 pacientes que não compareceram para revisão, portadores de lesão de Bankart com perda óssea glenóidea maior do que $25 \%$ ou lesão de Hill-Sachs com aproximadamente $25 \%$ ou mais de lesão da superfície da cabeça umeral. Outros tipos de instabilidade como atraumática, multidirecional, posterior e voluntária também foram excluídos, assim como pacientes com crises convulsivas sem controle. $\mathrm{O}$ critério de inclusão foi: instabilidade ântero-inferior traumática submetida a reparo da lesão de Bankart com uso de âncoras metálicas e sutura labial, com seguimento mínimo de dois anos.

Restaram 302 pacientes, correspondendo a 314 ombros. Os pacientes assinaram termo de concordância estabelecido pelo conselho de ética dos hospitais envolvidos com a pesquisa. Pertenciam a indivíduos do sexo masculino 274 ombros $(87,3 \%)$ e 40 aos do feminino $(12,7 \%)$. A média de idade no primeiro episódio da luxação ou subluxação foi de 23,04 \pm 8,03 anos (mínima de oito e máxima de 70 anos). A média de idade na época da cirurgia foi de 28,81 $\pm 10,14$ anos (mínima de 14 e máxima de 74 anos). O lado direito foi acometido em 186 pacientes $(61,6 \%)$ e o esquerdo, em $116(38,4 \%)$. Foram acometidos bilateralmente 12 pacientes $(4,0 \%)$. Foram praticadas 180 cirurgias no lado dominante $(57,6 \%)$ e 133 no lado não dominante $(42,4 \%)$.

Constou do questionário de avaliação o informe dado pelos pacientes quanto ao número aproximado de episódios de luxações e subluxações. Em 96 ombros ocorreram luxações, 116 sofreram subluxações e 102 apresentaram luxações e subluxações intercaladas. No grupo de ombros que luxaram, 83 apresentaram entre um e 10 episódios, seis entre 11 e 20, e sete, mais de 20 vezes. Entre os 116 que subluxaram, 68 tiveram entre um e 10 episódios; 15 entre 11 e 20; 28 relataram mais de 20 episódios.

Em relação à prática esportiva, identificamos: 93 ombros $(29,6 \%)$ pertenciam a não praticantes de esportes, 103 a desportistas de contato $(32,8 \%)$ e 118 $(37,6 \%)$ a praticantes de outros tipos de esportes. Entre os pacientes, $48(15,9 \%)$ apresentavam ao exame físico sinal de hiperelasticidade capsuloligamentar associada, caracterizada pela presença do sinal do sulco subacromial. Apresentavam histórico de convulsões nove pacientes (3\%). Todos estavam sob controle clínico à época da cirurgia. Entre os pacientes, 16 (5,3\%) apresentavam lesão do manguito rotador associada, 104 $(34,4 \%)$ eram portadores de lesão SLAP em associação, sendo 10 do tipo I $(9,6 \%), 61$ do tipo II $(58,7 \%)$, 29 do tipo III $(27,9 \%)$ e quatro do tipo IV $(3,8 \%)$; um paciente $(0,3 \%)$ foi operado para tratamento de recidiva de cirurgia aberta (capsuloplastia anterior).

Todos os pacientes foram caracterizados como portadores de instabilidade traumática devido à presença de lesão de Bankart, em associação ou não com a lesão de Hill-Sachs.

$\mathrm{Na}$ avaliação clínica foram mensuradas as amplitudes dos movimentos (ADM) comparando-se o ombro operado com o normal. Nos casos de cirurgias bilaterais, utilizamos os parâmetros médios das amplitudes do grupo em estudo como padrão. Os testes utilizados para pesquisa de instabilidade foram o sinal do sulco subacromial, teste da gaveta ântero-posterior, teste de recolocação e de apreensão(11).

A avaliação radiográfica foi feita através das incidências, ântero-posterior verdadeira (paciente inclinado $30^{\circ}$ sobre o lado a ser examinado e ombro em rotações neutra, externa e interna), acrescentando-se o perfil estrito da glenóide, de Bernageau ${ }^{(12)}$.

Como critérios de avaliação de resultados, utilizamos a classificação da UCLA (University of California at Los Angeles) modificado por Ellman ${ }^{(13)}$ e os critérios de Rowe et al ${ }^{(14)}$.

Ressonância magnética (RM) foi realizada em 36 ombros $(11,4 \%)$, artrorressonância magnética (artro- 
RM) em $73(23,2 \%)$ e tomografia computadorizada (TC) em seis $(1,9 \%)$.

Foram identificados 194 ombros $(61,8 \%)$ com lesão de Hill-Sachs e 54 (17,2\%) com lesão de Bankart óssea.

Na análise estatística utilizou-se um computador tipo PC. Para o cálculo das médias, medianas, desvios-padrão e tabelas 2 × 2, aplicou-se programa para análise estatística Epi Info versão 6.04d. Empregaram-se nos cálculos os testes $t$ de Student e exato de Fisher. Considerou-se como valor de significância estatística $\mathrm{p} \leq$ 0,05 .

\section{Técnica cirúrgica}

O procedimento é realizado com o paciente sob anestesia geral e posicionado em decúbito lateral, do lado oposto. Anestesia de bloqueio do plexo braquial é usada como complemento, visando a analgesia prolongada no pós-operatório imediato. São aplicadas trações vertical e longitudinal, mantendo-se o membro superior em abdução de aproximadamente $30^{\circ}$, flexão de $15^{\circ} \mathrm{e}$ inclinação dorsal do tronco de $30^{\circ}$. Usamos uma cânula posterior (CP) para irrigação e instrumentação, localizada $2,0 \mathrm{~cm}$ distais e a $2,0 \mathrm{~cm}$ mediais ao ângulo póstero-lateral do acrômio, correspondendo ao espaço entre os músculos infra-espinal e redondo menor. Uma cânula para artroscopia (CAS) é demarcada por visão intra-articular no intervalo dos rotadores, junto à borda anterior do músculo supra-espinal, em correspondência topográfica externa de aproximadamente $1,0 \mathrm{~cm}$ à frente do ângulo ântero-lateral do acrômio. Uma segunda cânula anterior é inserida, por visão intra-articular, no intervalo dos rotadores, junto à borda superior do tendão subescapular (CAI) (figura 1). Pelo portal posterior inserimos uma cânula de $6,0 \mathrm{~mm}$ de diâmetro, enquanto nos portais anteriores utilizamos cânulas de $8,25 \mathrm{~mm}$.

O procedimento inicia-se com a realização do inventário articular, com o artroscópio introduzido através do portal posterior.

Havendo lesão SLAP do tipo II ou dos tipos III e IV com desinserção labial, procede-se nesse momento à decorticação do contorno glenoidal superior e escarificação do lábio, deixando as interfaces cruentas e pre- paradas para a fixação de âncora e sutura, após sutura da lesão de Bankart.

Em seguida, transferimos o artroscópio para o portal ântero-superior. A instrumentação cirúrgica é realizada através do portal ântero-inferior e a irrigação passa a ser feita através do portal posterior (figura 1).

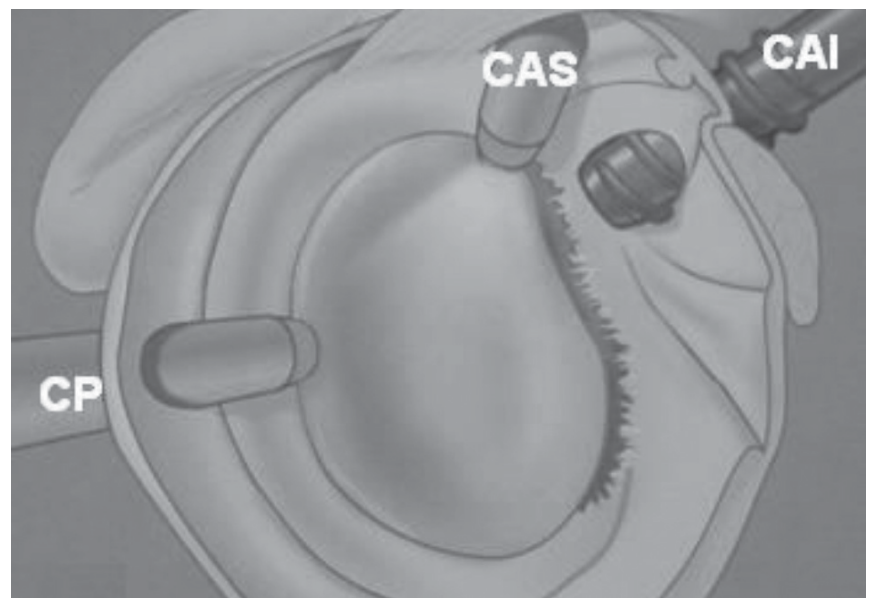

Figura 1 - Posição dos portais de artroscopia: CP (cânula posterior), CAS (cânula ântero-superior), CAI (cânula ântero-inferior).

Posteriormente, realizamos desbridamento na área da lesão de Bankart, com decorticação da borda anterior da glenóide e colo da escápula adjacente (figura 2). Realiza-se o desbridamento e regularização labial.

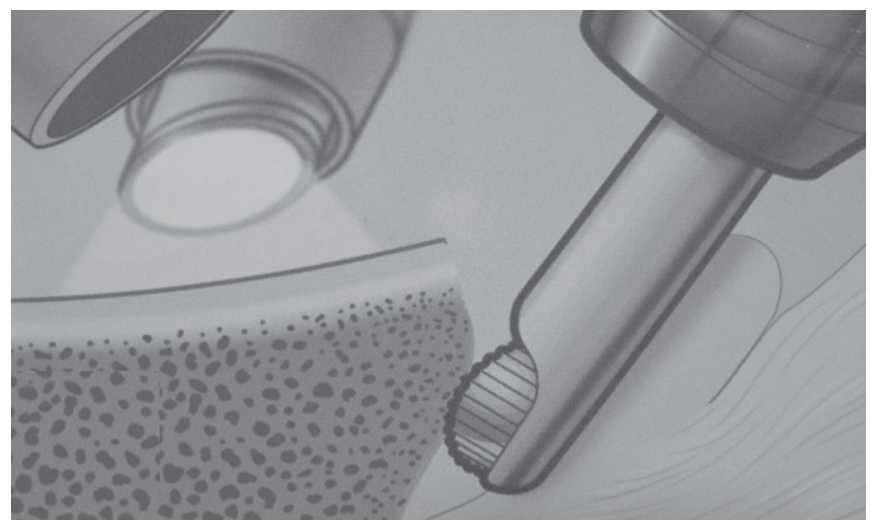

Figura 2 - Decorticação da borda anterior da glenóide

Demarcamos os pontos de inserção das miniâncoras de 2,7 x 5,0mm, fixadas a fios de sutura não-absorvíveis de $\mathrm{n}-2$, posicionados com inclinação de $45^{\circ} \mathrm{em}$ relação à superfície da glenóide. Os mesmos são separados entre si por aproximadamente $1,0 \mathrm{~cm}$ e situados na borda anterior ou avançando até $3,0 \mathrm{~mm}$ sobre a 
superfície articular. Utiliza-se um número mínimo de três ancoras (figura 3). Se comparado ao visor de um relógio, no ombro esquerdo as âncoras são inseridas nas posições 7, 9 e 11 horas, enquanto no ombro direito a equivalência se refere a 5, 3 e 1 hora.

Uma pinça tipo Caspari, ou rosqueada (suture hook), com fio monofilamentar $n^{\circ} 1$, é inserida através da cânula ântero-inferior e com a mesma transfixamos o lábio e ramo anterior do ligamento glenoumeral inferior (complexo labioligamentar ântero-inferior) em um ponto situado a aproximadamente $1,0 \mathrm{~cm}$ no sentido caudal, em relação à âncora mais distal, com a finali-

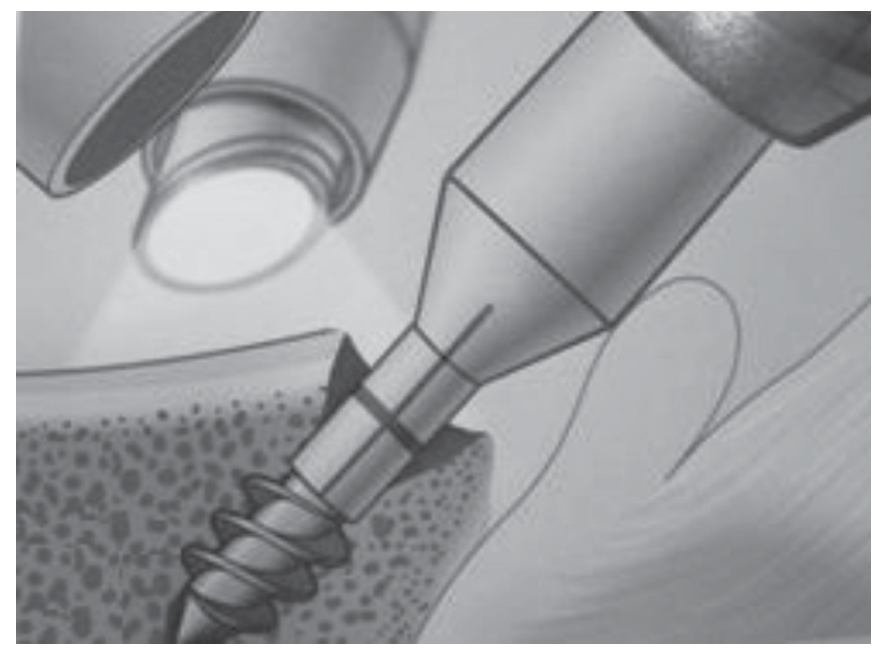

Figura 3 - Posicionamento e inserção da âncora. Observar ângulo de inclinação de aproximadamente $45^{\circ}$ e completo sepultamento da mesma

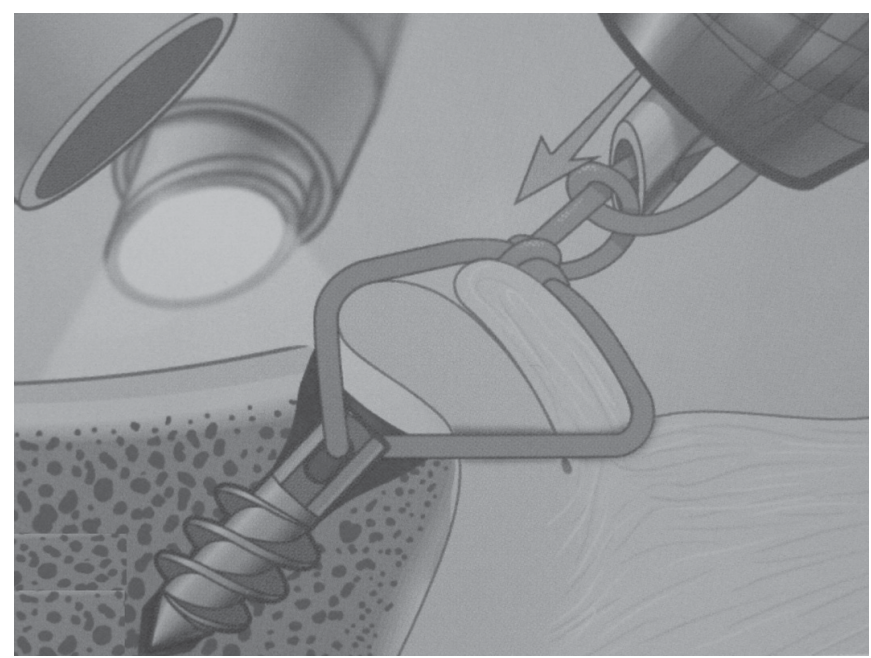

Figura 5a - Nó "revo" dade de produzir-se um efeito de retensionamento ligamentar no momento da sutura (figura 4). Nesse momento, a pinça conduz o fio monofilamentar (fio condutor), cuja extremidade será exteriorizada através do portal posterior, por onde será também exteriorizada uma das extremidades do fio não-absorvível preso à ancora. Os dois fios são atados um ao outro e o fio condutor é então tracionado ao longo do interior da cânula do portal ântero-inferior, até que o mesmo conduza o fio não-absorvível através do lábio. São

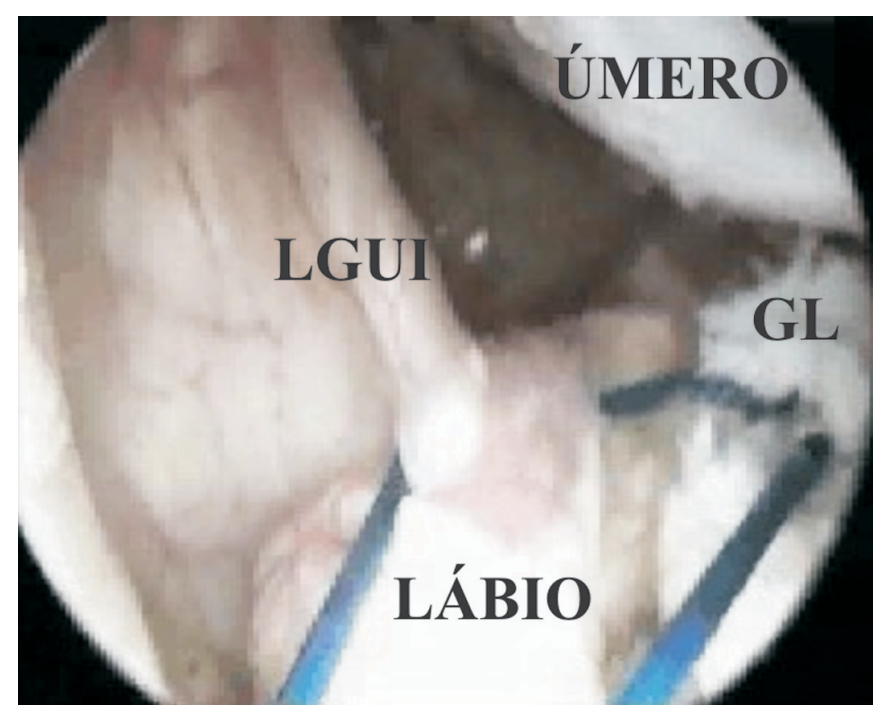

Figura 4 - Detalhe do retencionamento do complexo labioligamentar inferior. Ligamento glenoumeral inferior (LGUI), glenóide $(G L)$, cabeça umeral (úmero).

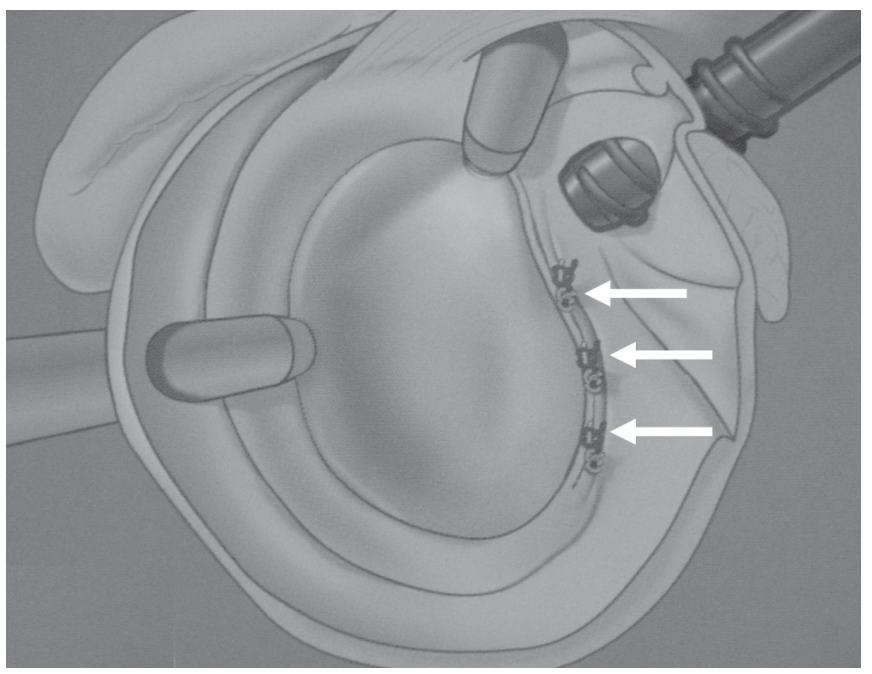

Figura 5b - Distribuição, preferencialmente eqüidistante, dos pontos de sutura labial (setas brancas) 
dados cinco nós intercalados (tipo "revo")(9) (figuras $5 a, 5 b)$.

Quando nos deparamos com um lábio muito danificado e/ou hipoplásico, utilizamos o reforço com a sutura adicional do ligamento glenoumeral médio superposto ao lábio. Se a lesão labial é representada por uma ALPSA lesion ${ }^{(15)}$, a abordagem deverá ser feita através de cuidadosa desinserção do lábio e recomposição anatômica do seu volume.

Na existência de uma lesão SLAP, fazemos o seu reparo após correção da lesão de Bankart de acordo com o tipo apresentado: desbridamento apenas, no tipo I; desbridamento e sutura, no tipo II; ressecção da alça de balde e fixação labial nos tipos III e IV. Havendo lesão associada do manguito rotador, o seu reparo é feito após realização dos procedimentos articulares.

Sutura dos portais de pele, curativos e imobilização em tipóia tipo Velpeau, durante 21 dias são os procedimentos seguintes. Programa de reabilitação é instituído após o 21ํ dia pós-cirúrgico.

\section{RESULTADOS}

Foram observadas 28 recidivas $(8,9 \%)$, todas operadas para tratamento de luxação. Sete pacientes $(2,2 \%)$ apresentaram apreensão residual.

Pela classificação segundo os critérios da UCLA, como relatado por Ellman et al ${ }^{(13)}$, foram excluídos os 28 ombros que tiveram recidivas $(8,9 \%)$, o que perfaz uma análise de 286 ombros. A variação observada na escala de Rowe, aplicada aos 314 ombros, oscilou de 25 a 100, com média de 91,8 $\pm 16,4$ pontos (tabela 1 ).

\section{TABELA 1}

Análise de resultados segundo os critérios da UCLA e Carter-Rowe

\begin{tabular}{lcccccc}
\hline & $\begin{array}{c}\text { Total } \\
\text { ombros }\end{array}$ & Excelentes & Bons & Regulares & Ruins \\
\hline UCLA & 286 & $217(75,9 \%)$ & $62(21,7 \%)$ & $5(1,7 \%)$ & $2(0,7 \%)$ \\
Rowe & 314 & $260(82,8 \%)$ & 20 & $(6,4 \%)$ & $11(3,5 \%)$ & $23(7,3 \%)$
\end{tabular}

No grupo estudado, 221 pacientes eram praticantes de esportes. O nível de retorno à prática esportiva está detalhado na tabela 2 .
TABELA 2

Retorno à prática de esportes

\begin{tabular}{cccc}
\hline Total & Mesmo nível & Nível inferior & Não retornaram \\
\hline 221 & $184(83,2 \%)$ & $18(8,1 \%)$ & $19(8,6 \%)$ \\
\hline
\end{tabular}

Osteoartrose pós-cirúrgica ocorreu em 15 pacientes $(4,8 \%)$ e foi classificada em três estágios, segundo Samilson e Prieto, como citado por Godinho ${ }^{(2)}$ (tabela 3).

TABELA 3

Incidência de osteoartrose pós-cirúrgica, segundo a classificação de Samilson e Prieto(20)

\begin{tabular}{cccc}
\hline Total & Estágio I & Estágio II & Estágio III \\
\hline $15(4,8 \%)$ & $7(2,2 \%)$ & $4(1,3 \%)$ & $4(1,3 \%)$ \\
\hline
\end{tabular}

$\mathrm{Na}$ análise estatística das recidivas observamos diferença significativa entre dois grupos de pacientes: os operados antes e após 1999 (tabela 4).

TABELA 4

Correlação entre recidivas e época da cirurgia foi de $p=0,013$

\begin{tabular}{cccc}
\hline Período & $\begin{array}{c}\text { № de } \\
\text { recidivas }\end{array}$ & Percentagem & $\begin{array}{c}\text { № total de } \\
\text { cirurgias }\end{array}$ \\
\hline 1996 a 1999 & 16 & $15 \%$ & 107 \\
2000 a 2005 & 12 & $5,8 \%$ & 207 \\
\hline
\end{tabular}

As tabelas 5 e 6 demonstram as correlações identificadas entre recidiva e: frouxidão capsuloligamentar ( $p$ $=0,16)$, convulsão $(\mathrm{p}=0,00039)$.

TABELA 5

Correlação entre frouxidão capsuloligamentar e recidiva $(p=0,16)$

\begin{tabular}{ccc}
\hline Total & Com recidiva & Sem recidiva \\
\hline 48 & $7(14,5 \%)$ & $41(85,5 \%)$ \\
\hline
\end{tabular}

TABELA 6

Correlação entre convulsão e recidiva $(p=0,00039$ )

\begin{tabular}{ccc}
\hline № total de pacientes & Com recidiva & Sem recidiva \\
\hline $9(2,9 \%)$ & $5(55,6 \%)$ & $4(44,4 \%)$ \\
\hline
\end{tabular}

Encontramos evidência estatisticamente significativa $(p=0,004)$ da relação direta entre recidiva e prática de esportes de contato (tabela 7). 
TABELA 7

Correlação entre esporte de contato e recidiva $(p=0,004)$

\begin{tabular}{ccc}
\hline № total de pacientes & Com recidiva & Sem recidiva \\
\hline $103(32,8 \%)$ & $12(11,7 \%)$ & $91(88,3 \%)$
\end{tabular}

Não identificamos no cruzamento estatístico - em ombros que apresentavam lesão de Hill-Sachs < 25\% da superfície da cabeça umeral $(60,19 \%)$ e ombros com lesão de Bankart ósseo > 325\% (17,19\%) - expressiva incidência de recidiva $(\mathrm{p}=0,62$ para Hill Sacks e $\mathrm{p}=$ 0,546 para Bankart) (tabelas 8 e 9).

TABELA 8

Correlação entre lesão de Hill-Sachs e recidiva $(p=0,62)$

\begin{tabular}{ccc}
\hline № total de ombros & Com recidiva & Sem recidiva \\
\hline $194(61,8 \%)$ & $19(9,8 \%)$ & $175(90,2 \%)$ \\
\hline
\end{tabular}

TABELA 9

Correlação entre lesão de Bankart e recidiva $(p=0,546)$

\begin{tabular}{ccc}
\hline № total de ombros & Com recidiva & Sem recidiva \\
\hline $54(17,2 \%)$ & $6(11,1 \%)$ & $48(88,9 \%)$ \\
\hline
\end{tabular}

Houve correlação estatisticamente válida entre índice de recidiva e reparo das lesões SLAP $(\mathrm{p}=0,015)$ (tabela 10).

TABELA 10

Correlação entre lesão SLAP e recidiva $(P=0,015)$

\begin{tabular}{ccc}
\hline № total de ombros & Com recidiva & Sem recidiva \\
\hline $104(33,2 \%)$ & $3(2,9 \%)$ & $101(97,1 \%)$ \\
\hline
\end{tabular}

\section{DISCUSSÃO}

Em uma análise crítica da literatura, observamos que alguns fatores ligados à grande variação de resultados dizem respeito ao tempo de seguimento ${ }^{(16)}$, experiência do cirurgião ${ }^{(17-18)}$, seleção de pacientes e técnica utilizada $^{(19)}$.

Dedicamo-nos à análise de resultados a longo prazo de uma técnica definida, um único tipo de material (âncoras metálicas) e todos os pacientes operados por dois cirurgiões experientes.
Neste estudo, foram utilizados dois critérios de avaliação de resultados: 1) UCLA pela sua simplicidade de execução e conseqüente facilidade de interpretação, somada à avaliação subjetiva dos próprios pacientes. Como critica, o método deixa a desejar por não incluir a análise de recidivas, sendo apenas um método de avaliação funcional ${ }^{(13)}$; 2) o método de Rowe, que objetiva especificamente a analise de resultados do tratamento da instabilidade do ombro ${ }^{(14)}$.

O uso de âncoras metálicas representa avanço histórico da técnica de artroscopia em nosso serviço, cujo inicio data de março de 1990. Passamos pela experiência inicial com uso da técnica de sutura translabial-transglenóide ${ }^{(7)}$, com índice de recidiva de 13,9\%, evoluindo para uso de tachas absorvíveis (Suretac ${ }^{\circledR}$ Smith \& Nephew Endoscopy, Andover, MA) ${ }^{(20)}$, com $10 \%$ de recidivas e âncoras do tipo arpão ${ }^{(20)}$.

Finalmente, passamos a utilizar os miniparafusos metálicos e, mais recentemente, âncoras absorvíveis.

$\mathrm{Na}$ análise estatística de recidivas podemos observar uma diferença significativa $(p=0,013)$ entre dois grupos de pacientes: aquele que vai de 1996 a 1999 e o outro, operado entre 2000 e 2005 . O primeiro grupo, além de constituir a primeira fase da curva de aprendizagem dos cirurgiões com o novo material, tinha no mesmo, deficiências sensíveis do ponto de vista de qualidade.

No segundo grupo (pacientes operados entre 2000 e 2005) passamos a utilizar material de última geração em termos de equipamentos, instrumental cirúrgico e âncoras, o que, associado à maior experiência dos cirurgiões, provavelmente possa explicar a redução estatisticamente significante $(\mathrm{p}=0,013)$ no percentual de recidivas.

Os resultados apresentados por Boileau et al em 91 pacientes operados e avaliados segundo os critérios de Rowe evidenciaram pontuação média de 77,8 pontos

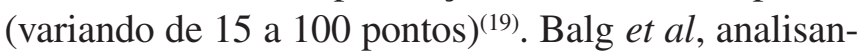
do os resultados de 131 pacientes operados e avaliados segundo o mesmo critério, encontraram media de $81,5$ pontos (10 a 100$)^{(21)}$.

Mishra et al avaliaram 42 pacientes operados com uso de âncoras e suturas ou tachas absorvíveis acrescentando radiofreqüência monopolar para obtenção de 
encurtamento dos ligamentos glenoumerais médio, ântero-inferior e póstero-inferior. Esses autores observaram média de 89,1 pontos utilizando classificação de Rowe modificada ${ }^{(22)}$. Nere et al, analisando 11 pacientes, encontraram índice de Rowe de 74,5 (variando de 35 a 100 pontos) $)^{(23)}$. Larrain et al descreveram $91,8 \%$ de resultados bons-excelentes segundo Rowe ${ }^{(24)}$. Marquardt et al, avaliando 54 pacientes, obtiveram melhora do índice de Rowe de 31,8 para 92,1. Segundo o mesmo critério, 43 pacientes $(81,1 \%)$ obtiveram resultado excelente; seis $(11,3 \%)$, bom resultado; um $(1,9 \%)$, resultado regular; e três $(5,7 \%)$, resultado ruim ${ }^{(25)}$. Gartsman et al obtiveram, em 53 pacientes operados, 49 resultados excelentes ou bons, segundo o índice de Rowe. Entretanto, a maioria desses pacientes foi tratada suplementarmente com capsuloplastia termal a laser, o que não foi usado em nossa casuística ${ }^{(26)}$.

Pela análise dos nossos resultados através do índice de Rowe, podemos observar que ocorreu variação de 25 a 100 pontos, com média de 91,8 . Chama atenção o fato de que o número de pacientes operados por via artroscópica por nós supera o número de casos descritos nos trabalhos da literatura consultada, exceto em estudos de metanálise.

Neri et al encontraram índice de resultados segundo a UCLA de 29,6 pontos (variando de 24 a 35), com resultados bons-excelentes em oito pacientes $(72,7 \%) \mathrm{e}$ razoáveis e ruins em três $(27,3 \%)^{(23)}$.

Observamos um índice de resultados segundo a UCLA variando de 19 a 35 pontos, com media de 33,8 pontos; 279 pacientes $(97,6 \%)$ obtiveram bons-excelentes resultados e sete $(2,4 \%)$, regulares e ruins. Em dois pacientes os resultados foram classificados como ruins, de acordo com a UCLA. O primeiro deles (ACM), masculino, operado com 25 anos de idade, apresentou lesão SLAP do tipo III e lesão de Bankart labial apenas. Relatava ter sofrido episódios de subluxações com mais de 20 ocorrências. Não apresentava lesão de Hill-Sachs e nem hiperelasticidade capsuloligamentar. Foram utilizadas cinco âncoras de fabricação nacional para reparo das lesões. $\mathrm{Na}$ avaliação final evidenciava artrose glenoumeral (Samilson III), pontuação da UCLA de 19 pontos e índice de Rowe de 60 pontos. Não se evidenciava claramente a exposição ou mau posicionamento das âncoras. Chama atenção o fato de que o paciente desenvolveu um quadro grave de capsulite adesiva pós-cirúrgico no primeiro mês pós-operado, evoluindo com rigidez acentuada e persistência de limitação dos movimentos até dois anos e meio, quando foi realizada nova cirurgia para desbridamento articular, capsulotomia circunferencial, liberação do intervalo dos rotadores e retirada da âncora mais distal, que mostrava sinais de exposição. Outro paciente, homem, operado aos 25 anos de idade, apresentou lesão de Bankart labial, lesão SLAP do tipo III e lesão parcial da face articular do tendão supra-espinal (lesão PASTA) associadas; foi submetido à fixação da lesão de Bankart com três âncoras e da lesão SLAP com duas âncoras. No pós-operatório imediato, o paciente apresentou dor intensa com limitação significativa das amplitudes dos movimentos evidenciadas a partir do segundo mês. Perdeu-se o controle do mesmo, que retornou para revisão um ano após a cirurgia, com persistência do quadro clínico e exame radiográfico evidenciando osteoartrose glenoumeral (Samilson II) secundária à exposição articular das âncoras, as quais foram retiradas na revisão cirúrgica.

Com relação à atividade esportiva, Boileau et $a l^{(19)}$ não encontraram correlação entre recidiva e o fato de o esporte ser ou não de contato. Balg e Boileau, em estudo de metanálise publicado posteriormente, identificaram como fatores de risco para a cirurgia artroscópica, no tratamento da instabilidade anterior, a prática de esportes de contato ou competitivos, ou aqueles com envolvimento do arremesso forçado ${ }^{(21)}$.

Burkhart et al e Simon et al citam a participação em esportes de contato ou colisão como fatores que influenciam no resultado cirúrgico ${ }^{(10,26)}$. O mesmo é confirmado por Hobby et al em estudo de revisão sistemática e metanálise de 62 publicações incluindo 3.044 cirurgias artroscópicas ${ }^{(27)}$. Marquardt et al, avaliando os resultados de 54 pacientes operados via artroscópica, dos quais 42 participavam de esportes, $36(85,7 \%)$ retornaram ao mesmo nível anterior em esporte de competição ${ }^{(25)}$. Larrain et al observaram que $84,3 \%$ dos pacientes operados retornaram ao mesmo nível do esporte num período médio de 7,5 meses pós-cirúrgicos, variando de 5,5 a nove meses ${ }^{(24)}$. Em nossa análise, dos 221 praticantes de esportes, $184(83,2 \%)$ retornaram ao mesmo nível, $18(8,1 \%)$ a um nível inferior e 
$19(8,6 \%)$ não retornaram à prática esportiva, o que iguala os nossos resultados com aqueles da literatura. Semelhante ao descrito por vários autores, há evidência estatística em nosso estudo $(p=0,004)$ da importância e correlação direta da prática de esporte de contato com as recidivas $(11,7 \%)^{(10,21,26-27)}$.

Boileau et al classificaram subjetivamente as lesões de Hill-Sachs como pequenas, médias e grandes por visão artroscópica. Em sua série foram avaliados $84 \%$ dos pacientes e as lesões de Hill-Sachs grandes apresentaram significância estatística para recidiva de luxações. Já para as lesões glenóideas do tipo Bankart com avulsão de fragmento ósseo, não houve significância estatística para recidiva, porém, quando se analisava o desgaste glenóideo, e este era superior a $25 \%$, a significância estava presente ${ }^{(19)}$.

Hobby et al relatam que a perda do contorno esclerótico da glenóide ântero-inferior na visão radiográfica em ântero-posterior é fator predisponente para recidiva da luxação. Nesses casos, o reparo artroscópico é contraindicado. Nesse mesmo artigo, os autores citam que a presença da lesão de Hill-Sachs na incidência em ântero-posterior com o braço em rotação externa é um fator que deve ser levado em consideração para recidiva ${ }^{(27)}$.

Marquardt et al, em sua série de 54 ombros avaliados por radiografias, excluíram aqueles com lesão de Bankart ósseo maior que $25 \%$ e lesão de Hill-Sachs maior que $30 \%$ para tratamento artroscópico, por considerá-los de alto risco de recidiva ${ }^{(25)}$. Kim et al encontraram taxa de recidiva aumentada para pacientes com defeito ósseo glenóideo maior que $30 \%$ da circunferência da glenóide ${ }^{(28)}$. Larrain et al afirmaram que os atletas apresentando lesões de Hill-Sachs maiores que $30 \%$ da cabeça umeral e defeito maior que $25 \%$ da extensão ântero-posterior da glenóide foram selecionados para se submeter a tratamento aberto pela técnica de Latarjet ${ }^{(24)}$. Burkhart et al relataram a importância da "lesão da pêra invertida" e da lesão de Hill-Sachs como critério de exclusão para artroscopia. Na série tratada artroscopicamente, obtiveram $70 \%$ de falhas ${ }^{(10)}$. Dos 314 ombros do nosso estudo, 189 $(60,19 \%)$ apresentavam lesão de Hill-Sachs e 54 $(17,19 \%)$ tinham lesão óssea de Bankart. Todos os ombros que apresentavam lesão de Bankart ósseo apre- sentavam também a lesão de Hill-Sachs. No cruzamento estatístico dessas lesões não houve significância em relação à recidiva $(\mathrm{p}=0,62$ para Hill-Sachs e $\mathrm{p}=0,546$ para Bankart). Os pacientes que apresentavam lesão de Hill-Sachs maior que $25 \%$ da superfície da cabeça umeral e lesão de Bankart ósseo ou desgaste glenóideo maior que $25 \%$ foram considerados dentro dos critérios de exclusão e tratados pela técnica de Latarjet-Patte, como descrita por Godinho ${ }^{(29)}$.

Estudos experimentais em biomecânica têm demonstrado que o complexo tendão-bicipital-lábio-superior tem importante papel na estabilização da articulação glenoumeral. Há clara e incisiva recomendação na literatura para que se faça a reparação das lesões SLAP em combinação com o reparo da lesão de Bankart $^{(30-35)}$.

No presente estudo, houve correlação estatisticamente válida $(\mathrm{p}=0,015)$ entre reparo das lesões SLAP e índice de recidivas, demonstrada pelo baixo percentual das mesmas, inferior à média geral $(2,9 \%$ e 8,9\%, respectivamente). Como não temos casos não reparados para análise de contraprova, podemos supor que o reparo associado das lesões SLAP representa um fator de proteção contra recidivas.

\section{CONCLUSÕES}

1) Há correlação estatisticamente identificada entre o índice de recidiva da instabilidade anterior traumática do ombro e:

a) convulsões no pós-operatório;

b) prática de esportes de contato;

c) presença das lesões de Bankart com Hill-Sachs caracterizadas por $25 \%$ ou mais de lesão óssea da glenóide ou da cabeça umeral, respectivamente.

2) Parece haver correlação entre o não reparo da lesão SLAP e o índice de recidiva da instabilidade anterior traumática do ombro.

\section{REFERÊNCIAS}

1. Johnson LL. Arthroscopy of the shoulder. Orthop Clin North Am. 1980;11(2):197-204.

2. Caspari RB. Shoulder arthroscopy: a review of the present state of the art. Contemp Orthop. 1982;4:523-31.

3. Morgan CD, Bodenstab AB. Arthroscopic Bankart suture repair: technique and early results. Arthroscopy. 1987;3(2): 111-22. 
4. Resch H, Povacz P, Wambacher M, Sperner G, Golser K. Arthroscopic extra-articular Bankart repair for the treatment of recurrent anterior shoulder dislocation. Arthroscopy. 1997; 13(2):188-200.

5. Caspari RB, Savoie FH. Arthroscopic reconstruction of the shoulder: The Bankart repair. In: McGinty JB et al., editors. Operative Arthroscopy. New York: Raven Press; 1991. p.507-15.

6. Carrera EF, Amatuzzi MM. Técnica artroscópica para correção da lesão de Bankart. Rev Bras Ortop. 1995;30(8):579-82.

7. Godinho GG, Souza JMG, Freitas JMA, Santos FML, Vieira AW, João FM. Tratamento da instabilidade anterior do ombro. Experiência com a técnica de Morgan. Rev Bras Ortop. 1997; 32(4):265-71.

8. Wolf EM. Arthoscopic capsulolabral repair using suture anchors. Orthop Clin North Am. 1993;24(1):59-69.

9. Snyder SJ, Strafford BB. Arthroscopic management of instability of the shoulder. Orthopedics. 1993;16(9):993-1002.

10. Burkhart SS, De Beer JF. Traumatic glenohumeral bone defects and their relationship to failure of arthroscopic Bankart repairs: significance of the inverted-pear glenoid and the humeral engaging Hill-Sachs lesion. Arthroscopy. 2000;16(7):677-94.

11. Rockwood CA Jr, Matsen FA III. Glenohumeral instability. In: Rockwood CA Jr, Matsen FA III. The Shoulder. Philadelphia: Saunders; 1998. p.611- 74.

12. Bernageau J. Le bourrelet glénoidien. Ann Radiol (Paris). 1995;38(5):266-74.

13. Ellman H, Hanker G, Bayer M. Repair of the rotator cuff. Endresult study of factors influencing reconstruction. J Bone Joint Surg Am. 1986;68(8):1136-44.

14. Rowe CR, Patel D, Southmayd WW. The Bankart procedure: a long-term end-result study. J Bone Joint Surg Am. 1978;60(1): $1-16$.

15. Neviaser NJ. The anterior labroligamentous periosteal sleeve avulsion lesion: a cause of anterior instability of the shoulder. Arthroscopy. 1993;9(1):17-21.

16. Manta JP, Organ S, Nirschl RP, Pettrone FA. Arthroscopic transglenoid suture capsulolabral repair. Five-year followup. Am J Sports Med. 1997;25(5):614-8.

17. Abrams JS. Arthroscopic approach to massive rotator cuff tears. Instr Course Lect. 2006;55:59-66.

18. Stein DA, Jazrawi L, Bartolozzi AR. Arthroscopic stabilization of anterior shoulder instability: a review of the literature. Arthroscopy. 2002;18(8):912-24.

19. Boileau P, Villalba M, Héry JY, Balg F, Ahrens P, Neyton L. Risk factors for recurrence of shoulder instability after arthroscopic Bankart repair. J Bone Joint Surg Am. 2006;88(8): 1755-63.

20. Godinho GG. Atualização em cirurgia do ombro.In: Pardini AG.Clínica ortopédica. Rio de Janeiro: Medsi; 2000. p. 203-306.

21. Balg F, Boileau P. The instability severity index score. A simple pre-operative score to select patients for arthroscopic or open shoulder stabilisation. J Bone Joint Surg Br. 2007;89(11):1470-7.

22. Mishra DK, Fanton GS. Two-year outcome of arthroscopic bankart repair and electrothermal-assisted capsulorrhaphy for recurrent traumatic anterior shoulder instability. Arthroscopy. 2001;17(8):844-9.

23. Neri BR, Tuckman DV, Bravman JT, Yim D, Sahajpal DT, Rokito AS. Arthroscopic revision of Bankart repair. J Shoulder Elbow Surg. 2007;16(4):419-24.

24. Larrain MV, Montenegro HJ, Mauas DM, Collazo CC, Pavón F. Arthroscopic management of traumatic anterior shoulder instability in collision athletes: analysis of 204 cases with a 4to 9-year follow-up and results with the suture anchor technique. Arthroscopy. 2006;22(12):1283-9.

25. Marquardt B, Witt KA, Liem D, Steinbeck J, Pötzl W. Arthroscopic Bankart repair in traumatic anterior shoulder instability using a suture anchor technique. Arthroscopy. 2006; 22(9):931-6. Comment in: Arthroscopy. 2007;23(6):684; author reply 684-5.

26. Roberts SN, Taylor DE, Brown JN, Hayes MG, Saies A. Open and arthroscopic techniques for the treatment of traumatic anterior shoulder instability in Australian rules football players. J Shoulder Elbow Surg. 1999;8(5):403-9.

27. Hobby J, Griffin D, Dunbar M, Boileau P. Is arthroscopic surgery for stabilisation of chronic shoulder instability as effective as open surgery? A systematic review and metaanalysis of 62 studies including 3044 arthroscopic operations. J Bone Joint Surg Br. 2007;89(9):1188-96.

28. Kim SH, Ha KI, Choy YB, Oh I. Arthroscopic anterior stabilization of the shoulder: two to six-year follow-up. J Bone Joint Surg Am 2003;85(8):1511-8.

29. Godinho GG. Cirurgia de Latarjet, modificada por Patte, para o tratamento da luxação recidivante anterior do ombro. Técnica e resultados (Dissertação). São Paulo: Universidade Federal de São Paulo. Escola Paulista de Medicina; 1999.

30. Snyder SJ, Karzel RP, Del Pizzo W, Ferkel RD, Friedman MJ. SLAP lesions of the shoulder. Arthroscopy. 1990;6(4):274-9.

31. Godinho GG, Freitas JMA, Leite LMB, Pina ERM. Lesões SLAP no ombro. Rev Bras Ortop. 1998;33(5):345-52.

32. Arciero RA, Taylor DC, Snyder RJ, Uhorchak JM. Arthroscopic bioabsorbable tack stabilization of initial anterior shoulder dislocations: a preliminary report. Arthroscopy. 1995;11(4):410-7.

33. Burkhart SS, Fox DL. Case report: arthroscopic repair of a type IV SLAP lesion - the red-on-white lesion as a component of anterior instability. Arthroscopy. 1993;9(5):488-92; discussion 493-7.

34. Rames RD, Karzel RP. Injuries to the glenoid labrum, including slap lesions. Orthop Clin North Am. 1993;24(1):45-53.

35. Warner JJ, Kann S, Marks P. Arthroscopic repair of combined Bankart and superior labral detachment anterior and posterior lesions: technique and preliminary results. Arthroscopy. 1994; 10(4):383-91.

Declaração de inexistência de conflitos de interesse: Declaramos para os devidos fins que não há qualquer conflito de interesse que impeça a publicação deste artigo. 